\title{
Diagnosis-related Groups (DRG) pricing and payment policy in China: where are we?
}

\author{
Lihua Yu, Jingjing Lang \\ Healthcare Cost and Price Division, China National Health Development Research Center of National Health Commission, Beijing, China \\ Correspondence to: Lihua Yu. Research Fellow, Deputy Director of Healthcare Cost and Price Division, China National Health Development Research \\ Center of National Health Commission, No. 9 Chegongzhuang Avenue, Xicheng District, Beijing 100044, China. Email: yulihua8888@163.com.
}

Submitted Nov 12, 2020. Accepted for publication Nov 22, 2020.

doi: $10.21037 / \mathrm{hbsn}-2020-8$

View this article at: http://dx.doi.org/10.21037/hbsn-2020-8

Diagnosis-related Groups (DRG) is an internationally recognized advanced hospital management method. It was pioneered by Yale University in the late 1960s and has been used as the inpatient prospective payment system by the U.S. Medicare program since 1983 (1,2). It has since been introduced in Australia, Germany, France, the UK, Japan, South Korea, and Scandinavia. It is widely used in the fields of pricing and payment, budget allocation, performance evaluation, and comparison (2). As a refined hospital management tool, DRG combined with the corresponding payment mechanism can effectively meet the requirements of health expenditure control, hospital management, and health insurance management (3). DRG is also crucial for promoting the shift of payment methods from retrospective payment to prospective payment and for improving the performance management of the healthcare system.

\section{The DRG concept}

DRG divides inpatients into a certain number of disease groups according to the severity of the diseases, the complexity of the treatment methods, and the homogeneity of the resource consumption to achieve a variety management goals $(1,2)$. In principle, DRG should cover all hospitalized patients, except for special patients with rare diseases, mental illness, or those treated with special regimens, such as Traditional Chinese Medicine, cutting-edge technology etc. The most important factors in determining which group a patient is assigned to is the principal diagnosis and principal procedure. Comorbidities and complications, length of stay, age, and other factors should also be considered. Hospitalization diseases can generally be divided into 600 to 2,000 disease groups (2). A country or a region can use DRG as a tool to conduct pricing and payment management, budget allocation, hospital performance evaluation, and comparison.

\section{Introduction of DRG versions to China}

In China, Beijing initially took the lead in DRG research by piloting a program in 2003. Since then, Zhejiang Province, Yunnan Province, Shenyang Province, Shanghai Municipal Province, and other cities have developed their respective local DRG versions and have actively piloted programs in the fields of payment and hospital performance evaluation.

The Chinese government mandated the inception of DRG piloting at the national level in 2017. At present, there are three main versions at the national level. The first version implemented was the C-DRG, which is used for pricing and payment. In 2017, the National Health Commission launched the C-DRG, comprising a total of 900 DRG groups, to reform pricing and payment in the cities of Sanming, Shenzhen, and Karamay, and three piloting hospitals in Fujian Province (3). The C-DRG has three primary characteristics. First, its DRG groups patients mainly according to clinical similarity, verified by the expenditure and cost data of more than 1,400 medical institutions reported in the "National Healthcare Cost and Price Monitoring and Research Network" which was established by the Finance Department of National Health Commission. Second, to facilitate hospital management, the C-DRG innovatively uses two DRG grouping tools, "Chinese Clinical Disease Terminology" (CCDT) and "Chinese Classification of Health Interventions" (CCHI) 
by mapping these tools to the International Classification of Disease (ICD)-10 and ICD-9-CM-3. Third, the C-DRG uses DRG groups as the unit to charge patients in all hospitals with a standard price, which is in turn paid via health insurance; this system represents the first successful reform of healthcare payment in China (3).

The second version of DRG which has been implemented in China is the CHS-DRG, which is used for health insurance payment. In 2019, the National Healthcare Security Administration launched the DRG payment reform in 30 piloting cities across the country, using the CHS-DRG which comprises a total of 376 adjacent-DRG groups and 618 DRG subgroups. The CHS-DRG uses the ICD-10 and ICD-9-CM-3 (National Healthcare Security Version) as its the grouping tools. The National Healthcare Security Administration proposed a DRG payment reform to conduct simulated piloting in 30 cities in 2020 and actual piloting in 2021.

The third version that has been employed in China is the CN-DRG, which is used for hospital performance evaluation and administration. In 2013, the Bureau of Medical Administration of National Health Commission launched the DRG on hospital performance evaluation, using the CN-DRG which comprises a total of 804 DRG groups and the ICD-10 and ICD-9-CM-3 (National Clinical Version 2.0) as grouping tools. The performance evaluation scope covers nearly 1,000 hospitals across 29 provinces and municipalities. In 2019, the CN-DRG was also introduced for indicator design in the performance examination of tertiary public hospitals.

\section{DRG pricing and payment reform}

For an extended period of time, healthcare payment in China has consisted of a fee-for-service payment method. In this system, the hospital is reimbursed by health insurance for each individual health care service provided and, to provide each service, the hospital is permitted to bill purchasers for all expenditures incurred $(3,4)$. The payment is directly related to the number of services, and thus the hospital is incentivized to increase the number of overall services during a patient's hospitalization (4).

The DRG pricing and payment reform uses the DRG group as the unit of pricing. With this system, the price of the service that hospitals bill purchasers is predetermined. The price of each group includes the healthcare services, drugs, and medical consumables provided from admission to discharge. The hospital is reimbursed by health insurance according to the DRG price and the reimbursement ratio. The hospital also bills the patient according to the same price and the out-of-pocket payment ratio. Which DRG group each patient is assigned to is closely related to the principal diagnosis and principal procedure during the hospitalization, and is not related to the number health care services received, or drugs or medical consumables used. The characteristic of DRG pricing and payment reform is that the price of each DRG group is determined in advance. All costs related to services, drugs, and consumables are to be borne by the hospital. The basic billing principle of DRG reform is that "excess expenditures will no longer be reimbursed and the balance will be retained". Therefore, the reform will emphatically promote the transformation of the Chinese hospital operation model from a focus on expanding revenue to a focus on cost control and optimization. It will also motivate hospitals to standardize diagnosis and treatment, improve efficiency, and ensure quality and safety.

An example is perhaps useful to illustrate the reformed process and its intended effects: a group of patients are diagnosed with primary hepatocellular carcinoma with comorbidities and undergo radical lobectomy as treatment during the hospitalization. Under the fee-for-service payment system, the expenditures for hospitalization range between 40,000 and 100,000 CNY for different patients according to the healthcare services, drugs, and medical consumables provided. Under the reformed DRG pricing and payment system, these patients would be classified into the DRG group named "major liver procedures with comorbidities and complications". Taking a piloting city as an example, the price of this DRG group would $80,000 \mathrm{CNY}$, and patients assigned to this DRG group would be billed at $80,000 \mathrm{CNY}$ regardless of the services, drugs, and consumables received. In providing these to the patient, the hospital would need to greatly refine their management of resources, standardize clinicians' diagnosis and treatment, and control costs to ensure quality while keeping expenditures low.

\section{Prospects for future applications}

China has intensified the degree of reform to its healthcare system in recent years, with the efforts to transform healthcare payment increasing each year and yielding remarkable success. According to the international experiences of DRG application and the current healthcare status of China, the future measures of DRG 
reform should include the following: first, a unified DRG grouping regulation and unified grouping tools for disease and health intervention classification on the national level should be published. Regardless to which field the DRG is applied, the version should be sufficiently consistent to facilitate comparisons between hospitals using standard terms and criteria. Second, a nationally unified monitoring system based on minimal DRG data should be established to monitor the diagnosis and treatment behaviors. This would aid in minimizing the risks of health insurance funding, and provide an information platform for conducting interhospital and interspecialty comparative analysis. Third, a long-term dynamic mechanism should be developed to regularly update DRG grouping, grouping tools, payment standards, and supporting policies. Fourth, multiple payment methods based on the characteristics of different healthcare services should be supported. Finally, it should be emphasized that clinicians are the linchpin in the revolution of healthcare payment. As all payment methods need to follow clinical norms, the classifications and terminologies of diagnosis and procedures should be unified at the national level. It is the responsibility of clinicians to effectively standardize and adopt sound diagnosis and treatment practices, as these form the foundation on which the future pricing and payment standards will be built.

\section{Acknowledgments}

Funding: None.

\section{Footnote}

Provenance and Peer Review: This article was commissioned by the editorial office of Hepatobiliary Surgery and Nutrition. The article did not undergo external peer review.

Cite this article as: Yu L, Lang J. Diagnosis-related Groups (DRG) pricing and payment policy in China: where are we? HepatoBiliary Surg Nutr 2020;9(6):771-773. doi: 10.21037/ hbsn-2020-8
Conflicts of Interest: Both authors have completed the ICMJE uniform disclosure form (available at http://dx.doi. org/10.21037/hbsn-2020-8). The authors have no conflicts of interest to declare.

Ethical Statement: The authors are accountable for all aspects of the work in ensuring that questions related to the accuracy or integrity of any part of the work are appropriately investigated and resolved.

Open Access Statement: This is an Open Access article distributed in accordance with the Creative Commons Attribution-NonCommercial-NoDerivs 4.0 International License (CC BY-NC-ND 4.0), which permits the noncommercial replication and distribution of the article with the strict proviso that no changes or edits are made and the original work is properly cited (including links to both the formal publication through the relevant DOI and the license). See: https://creativecommons.org/licenses/by-nc-nd/4.0/.

\section{References}

1. Fetter RB, Shin Y, Freeman JL, et al. Case mix definition by diagnosis-related groups. Med Care 1980;18:iii, 1-53.

2. Busse R, Geissler A, Quentin W, et al. Diagnosis-related groups in Europe: moving towards transparency, efficiency and quality in hospitals. Maidenhead: Open University Press, 2011:3-41.

3. Zhang ZZ, Jiang Q, Yu LH. The overall design for the pricing and payment regulation on Chinese Diagnosis Related Groups. Chinese Health Economics 2017;36:5-8.

4. Langenbrunner JC, Cashin C, O'Dougherty S. Designing and implementing health care provider payment systems: how-to manual. Washington DC: The World Bank, 2009:6-7.

(English Language Editor: J. Gray) 